\title{
Interpretation of the Cyclic Balmer Progression Phenomenon
}

\author{
Ryuko Hirata
}

Department of Astronomy, Kyoto University, Kyoto 606-8502, Japan

\author{
Atsuo T. Okazaki \\ Faculty of Engineering, Hokkai-Gakuen University, Toyohira-ku, \\ Sapporo 062-8605, Japan
}

\begin{abstract}
We have computed the Balmer-line profiles for the onearmed oscillation model and found that the model can naturally account for the cyclic Balmer progression phenomenon observed in the $V / R$ variable shell stars.
\end{abstract}

\section{What is the Balmer Progression?}

The Balmer progression in $\mathrm{V} / \mathrm{R}$ variable shell stars is characterized by the progressive radial velocity shift in Balmer shell lines towards the higher members. The radial velocity $v_{n}$ of the $\mathrm{H} n$ line varies as $d v_{n} / d n<0$ for $v_{n}<0$ (negative progression) and $d v_{n} / d n>0$ for $v_{n}>0$ (positive progression), accompanied by line asymmetry. In the lower members, the $V / R$ emission variation is observed in the sense that $V / R>1$ with positive progression and vice versa. Figure 1 shows the radial velocity variations in $\zeta$ Tau (Delplace 1970) and $48 \mathrm{Lib}$ (Aydin $\&$ Faraggiana 1978) against time and $n$. The stellar velocity is set to zero by correcting the orbital motion and the $\gamma$ velocity in the case of $\zeta$ Tau.

Table 1. Stellar and disk parameters adopted for $\zeta$ Tau and $48 \mathrm{Lib}$.

\begin{tabular}{|c|c|c|c|c|c|c|}
\hline & Star & & & Disk & & \\
\hline & $\zeta$ Tau & $48 \mathrm{Lib}$ & & $\zeta$ Tau & $48 \mathrm{Lib}$ & rem \\
\hline $\bar{M} / M_{\odot}$ & 215.0 & $\begin{array}{l}8.8 \\
\end{array}$ & $T_{\text {disk }}$ & 16700 & $\overline{9500}$ & \\
\hline$T_{\text {eff }}$ & 25000 & 18000 & $\alpha$ & 3.5 & 3.5 & (1) \\
\hline$R_{*} / R_{\odot}$ & 5.5 & 5.1 & $\delta$ & $0.5,0.95$ & $0.5,0.95$ & (2) \\
\hline$V_{\mathrm{e}} \sin i$ & 220 & 395 & $R_{\text {out }} / R_{*}$ & 23.5 & $\infty$ & (3) \\
\hline & $70^{\circ}, 82^{\circ}$ & $70^{\circ}, 82^{\circ}$ & $R_{\mathrm{em}} / R_{*}$ & 23.5 & 20 & (4) \\
\hline
\end{tabular}

Notes: (1) density gradient index. The equatorial density in the unperturbed disk varies as $r^{-\alpha}$. (2) perturbation amplitude, (3) disk size, and (4) size of emitting region. 


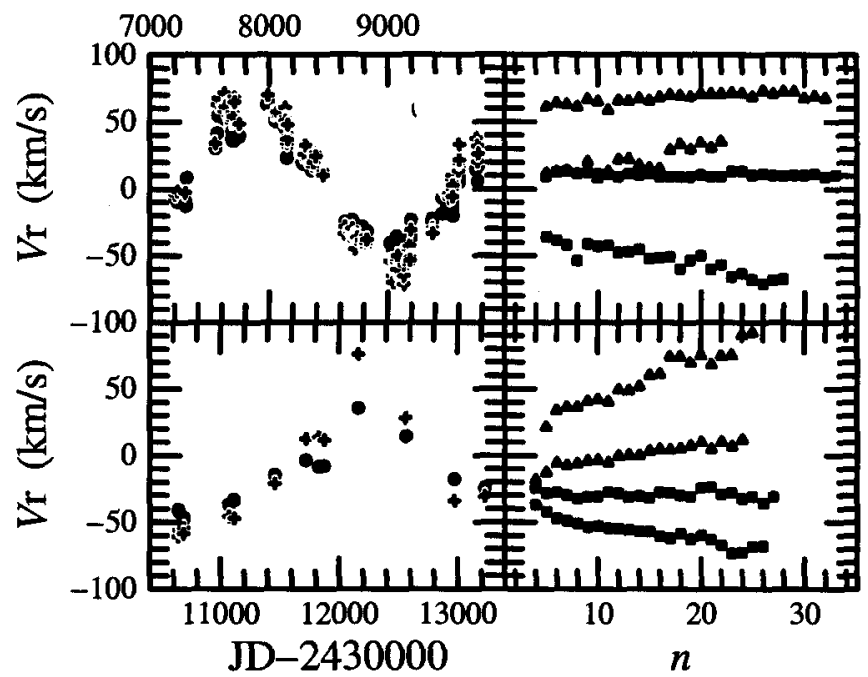

Figure 1. Observed Balmer progression in $\zeta$ Tau (top) and $48 \mathrm{Lib}$ (bottom). The mean radial velocities for $\mathrm{H} 5-7$ (filled circle) and H19-21 (plus sign) are plotted against time in the left panels, while, in the right panels, the individual radial velocities are plotted against $n$ for some typical epochs.

\section{Interpretation}

Hirata (1982) suggested that such cyclic progression can be explained in terms of the precessing elongated disk (see also Hirata \& Kogure 1984). The progression occurs when the line-of-sight velocity with maximum area of the stellar disk screened by the envelope differs from that with maximum optical thickness (absorption process). Later, Okazaki (1991) showed that the characteristics of the $V / R$ variation are well reproduced by the one-armed oscillation of a near Keplerian disk, a dynamical interpretation of the elongated disk. From the resemblance of the velocity field in these two models, it is expected that the cyclic Balmer progression is also generated in the latter model.

\section{Disk Model}

We have computed Balmer line profiles from disks with $m=1$ perturbation patterns. The assumptions we have made to construct model and compute the line profiles are:

- the disk is isothermal.

- the disk is inviscid. No advective motion is present in the unperturbed state. 


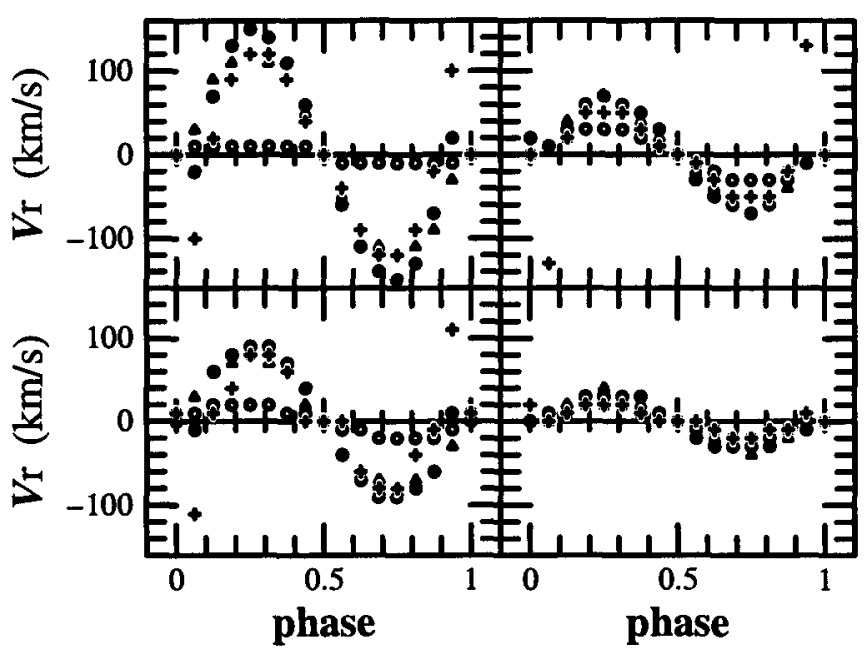

Figure 2. Simulated Balmer progression in $\zeta$ Tau (top) and $48 \mathrm{Lib}$ (bottom) for $\delta=0.95$ and with $i=70^{\circ}$ (left) and $i=82^{\circ}$ (right). The radial velocities are plotted against phase for $\mathrm{H} \alpha$ (open circle), $\mathrm{H} \beta$ (open triangle), $\mathrm{H} 7$ (filled circle) and H17 (plus sign).

- the density perturbation pattern is similar to the linear, isothermal $m=1$ eigenmode.

- we have assumed $b_{2}=1 / W$, where $b_{2}$ is the deviation factor from the LTE state for the second energy level of hydrogen and $W$, the dilution factor.

Basic stellar and disk parameters we have adopted are given in Table 1.

\section{Results and Discussion}

Radial velocities computed for the model described above are plotted against phase in Figure 2. We see that the one-armed oscillation model can naturally account for the observed progression. From close inspection of the simulated profiles, we notice:

1) The maximum absorption for $i=82^{\circ}$ occurs when the denser part is located in front of the star, in accordance with the observations of $\zeta$ Tau. On the other hand, the maximum absorption occurs at phases of 0.25 and 0.75 for $i=70^{\circ}$. This reflects the fact that the signs of density perturbation are opposite in the inner and outer parts of the disk, and the shell absorption is formed in the outer part for larger $i$ and in the inner to middle part for smaller $i$.

2) The relation between the radial velocities and the line asymmetry depends on the inclination angle $i$. For $i=82^{\circ}$, there appear a blue-winged, 
blue-shifted profile and then a red-winged, red-shifted profile as the phase proceeds, whereas for $i=70^{\circ}$, a blue-winged, red-shifted profile and a red-winged, blue-shifted profile appear.

The observed general trend is not clear on the latter feature at present. However, we can point out that $48 \mathrm{Lib}$ exhibited the asymmetry corresponding to our $i=82^{\circ}$ case in the ascending phase of the radial velocity around its maximum, while in the descending phase, the asymmetry like our $i=70^{\circ}$ case was observed in 1938-1944 and in 1970-1976 (Merill \& Sanford 1944; Aydin \& Faraggiana 1978).

In our simulations, we have adopted $b_{2}=1 / W$. It turned out that the resulting profiles are sensitive to the NLTE state, i.e., the $b_{2}$ factor. For example, no progression occurs when the LTE population $\left(b_{2}=1\right)$ is adopted. It is required to take into account the NLTE effect more exactly for the detailed comparison with the observation. Since the observed amplitude of the velocity variation is quite large in $\zeta$ Tau and $48 \mathrm{Lib}\left( \pm 80 \mathrm{kms}^{-1}\right)$, nonlinear simulations of the perturbed disks are also desirable. Nevertheless, the one-armed oscillation model has great potential to explain the complex progression phenomenon.

\section{References}

Aydin, C., Faraggiana, R. 1978, A\&AS 34, 51

Delplace, A.M. 1970, A\&A 7, 68

Hirata, R. 1982, in IAU Symp. 98, Be Stars, M.Jaschek, H.-G.Groth, Eds., Reidel, Dordrecht, p. 497

Hirata, R., Kogure, T. 1984, BAS India 12, 109

Merrill, P.W., Sanford, R.F. 1944, ApJ, 100, 14

Okazaki, A.T. 1991, PASJ, 43, 75 